\title{
AC SUSCEPTIBILITY MEASUREMENTS IN THE INVESTIGATION OF AGGREGATION IN MAGNETIC FLUIDS
}

\author{
P.C. FANNIN ${ }^{a}$ AND S.W. ChaRleS ${ }^{b}$ \\ ${ }^{a}$ Dept. of Electronic \& Electrical Engineering, Trinity College \\ Dublin 2, Ireland \\ ${ }^{b}$ Dept. of Chemistry, University College of North Wales, Bangor, LL57 2UW, UK
}

Measurements of the complex, frequency dependent susceptibility, $\chi(\omega)=\chi^{\prime}(\omega)-\mathrm{i} \chi^{\prime \prime}(\omega)$ of two water based magnetic fluids, over the frequency range $10 \mathrm{~Hz}$ to $1 \mathrm{MHz}$ are reported. Plots of the susceptibility components against $\log [f(\mathrm{~Hz})]$ are found to have approximate Debye-type profiles with the presence of Brownian relaxation being indicated by the frequency, $f_{\max }$, of the maximum of the loss-peaks in the $\chi^{\prime \prime}(\omega)$ profiles. Corresponding calculations of particle hydrodynamic radius indicate the presence of aggregation. Frohlich, normal and lognormal distribution functions are used to obtain an estimate of the aggregate size distribution in the samples.

PACS numbers: $75.50 . \mathrm{Tt}, 76.20 .+\mathrm{q}$

\section{Introduction}

Ferrofluids are colloidal suspensions of ultrafine single-domain particles of ferromagnetic or ferrimagnetic materials dispersed in a carrier liquid and stabilised by a suitable organic surfactant. The particles have radii ranging from approximately $2-10 \mathrm{~nm}$ and as they are single-domain, they are considered to be in a state of uniform magnetisation with magnetic moment, $m$, given by

$$
m=M_{\mathrm{s}} v,
$$

where $M_{\mathrm{s}}\left(\mathrm{Wb} / \mathrm{m}^{2}\right)$ denotes saturation magnetisation and $v$ is the magnetic volume of the particle. The magnetic moment of each particle has a preferred orientation (easy axis) relative to the particle due to magnetic anisotropy, $K$, which generally arises from a combination of shape and magnetocrystalline anisotropy.

The magnetic moment may relax either by rotational Brownian motion of the particle within the carrier liquid, with its magnetic moment locked in an axis of easy magnetisation, or by rotation within the particle. The time associated with the rotational diffusion is the Brownian relaxation time $\tau_{\mathbf{B}}$ [1]

$$
\tau_{\mathrm{B}}=3 V \eta / k T
$$


where $V$ is the hydrodynamic volume of the particle and $\eta$ is the dynamic viscosity of the carrier liquid. In the case of the second relaxation mechanism, the magnetic moment may reverse direction within the particle by overcoming an energy barrier, which for uniaxial anisotropy, is given by $K v$. This reversal time is referred to as the Néel relaxation time $\tau_{\mathrm{N}}$ and may be given in terms of Brown's [2] expressions for high and low barrier heights.

A distribution of particle sizes implies the existence of a distribution of relaxation times, with both relaxation mechanisms contributing to the magnetisation. They do so with an effective relaxation time $\tau_{\text {eff }}$, where

$$
\tau_{\text {eff }}=\tau_{\mathrm{N}} \tau_{\mathrm{B}} /\left(\tau_{\mathrm{N}}+\tau_{\mathrm{B}}\right)
$$

\section{Complex susceptibility}

The complex susceptibility $\chi(\omega)$, may be written in terms of its real and imaginary components

$$
\chi(\omega)=\chi^{\prime}(\omega)-\mathrm{i} \chi^{\prime \prime}(\omega)
$$

The theory developed by Debye [3] to account for the anomalous dielectric dispersion in dipolar fluids may be used to account for the analogous case of magnetic fluids [4]. According to Debye's theory, $\chi(\omega)$ has a frequency dependence given by the equation

where

$$
\chi(\omega)-\chi_{\infty}=\left(\chi_{0}-\chi_{\infty}\right) /(1+\mathrm{i} \omega \tau)
$$

$$
\tau=1 / \omega_{\max }=1 / 2 \pi f_{\max } .
$$

$f_{\max }$ is the frequency at which $\chi^{\prime \prime}(\omega)$ is a maximum, and $\chi_{0}$ and $\chi_{\infty}$ indicate susceptibility values at $\omega=0$ and at very high frequencies. $\chi(\omega)$ may also be expressed in terms of a logarithmic distribution function, $G(\ln \tau)$ [5] in the form

$$
\chi(\omega)=\chi_{\infty}+\left(\chi_{0}-\chi_{\infty}\right) \int_{0}^{\infty} G(\ln \tau) \mathrm{d} \ln \tau /(1+\mathrm{i} \omega \tau) .
$$

For the Frohlich [5] distribution function it is assumed that the distribution of relaxation times is limited within a certain range from $\tau_{1}$ to $\tau_{2}$

$$
G(\ln \tau)=1 / \ln \left(\tau_{1} / \tau_{2}\right), \quad \tau_{2}<\tau<\tau_{1}, \quad G(\ln \tau)=0, \quad \tau<\tau_{2} \text { or } \tau>\tau_{1}
$$

and, as $\tau_{1}$ and $\tau_{2}$ are proportional to the cube of the maximum and minimum hydrodynamic radii respectively, then

$$
r_{\max } / r_{\min }=\left(\tau_{1} / \tau_{2}\right)^{1 / 3}
$$

It is readily shown that [5]

and

$$
\begin{aligned}
& \chi^{\prime}(\omega)=\chi_{\infty} \\
& \quad+\left(\chi_{0}-\chi_{\infty}\right)\left[1 / \ln \left(\tau_{1} / \tau_{2}\right)\right] \ln \left(\tau_{1} \sqrt{1+\omega^{2} \tau_{2}^{2}} / \tau_{2} \sqrt{1+\omega^{2} \tau_{1}^{2}}\right)
\end{aligned}
$$

$$
\chi^{\prime \prime}(\omega)=+\left(\chi_{0}-\chi_{\infty}\right) 1 / \ln \left(\tau_{1} / \tau_{2}\right) \arctan \left(\omega \tau_{1}\right)-\arctan \left(\omega \tau_{2}\right)
$$

The values of $\tau_{1}$ and $\tau_{2}$ can be determined by means of fitting Eqs. (10) and (11) to the measured susceptibility data. The normal and lognormal distributions have the well-known form [6]. 


\section{Results and discussion}

The frequency-dependent complex susceptibility of two ferrofluid samples, namely fluids 1 and 2, was measured by means of the torcidal technique [7] over the frequency range $10 \mathrm{~Hz}$ to $1 \mathrm{MHz}$. The two fluids consisted of suspensions of magnetite in water with corresponding saturation magnetisations of $107 \mathrm{Gs}$ and $90 \mathrm{Gs}$ and mean magnetic particle radii, as determined by electron micrography, of $7 \mathrm{~nm}$ and $6 \mathrm{~nm}$ respectively. The plots of $\chi^{\prime}(\omega)$ and $\chi^{\prime \prime}(\omega)$ against $\log [f(\mathrm{~Hz})]$ for the fluids are found to have a Debye-type profile with a maximum of $\chi^{\prime \prime}(\omega)$ occurring at a frequency of approximately $f_{\max }=1 \mathrm{kHz}$ for fluid 1 and at $1.6 \mathrm{kHz}$ for fluid 2. From Eq. (2) and using a viscosity of $10^{-4} \mathrm{~N} \mathrm{~s} \mathrm{~m}^{-2}$, corresponding hydrodynamic radii $\left(r_{\mathrm{H}}\right)$ of $80 \mathrm{~nm}$ and $69 \mathrm{~nm}$ are determined. Even allowing for surfactant thickness (say $2 \mathrm{~nm}$ ) the hydrodynamic radii are greater than the sum of the magnetic radii plus surfactant thickness and is indicative of the existence of aggregation. To obtain a measure of the distribution of aggregates the susceptibility profiles were fitted to three distribution functions, namely the Frohlich, normal and lognormal distributions. The Frohlich distribution function gave an indication of the range of relaxation times, $\tau_{1}$ to $\tau_{2}$ from which corresponding values of radii, $r_{\max }$ to $r_{\min }$ were obtained. In the case of normal and lognormal distribution

\section{TABLE}

Experimental and fitted data for fluids 1 and 2.

\begin{tabular}{c|c|c}
\hline \hline Fluid & 1 & 2 \\
\hline$f_{\max }(\mathrm{Hz})$ & 1000 & 1600 \\
$\tau_{\max }(\mathrm{s})$ & $1.6 \times 10^{-4}$ & $9.95 \times 10^{-5}$ \\
$\left(1 / 2 \pi f_{\max }\right)$ & & \\
$r_{\mathrm{H}}(\mathrm{nm})$ & 80 & 69 \\
$\sigma_{\mathrm{n}}$ & 0.63 & 0.43 \\
$\sigma_{\mathrm{L}}$ & 0.76 & 0.52 \\
$\tau_{1}(\mathrm{~s})$ & $1.6 \times 10^{-3}$ & $6 \times 10^{-4}$ \\
$\tau_{2}(\mathrm{~s})$ & $1.5 \times 10^{-5}$ & $1.4 \times 10^{-5}$ \\
\hline \multicolumn{3}{|c}{$r_{\min }$ to $r_{\max }$} \\
\hline Frohlich (nm) & 37 to 174 & 36 to 125 \\
Normal & 30 to 130 & 39 to 125 \\
Lognormal & 28 to 128 & 36 to 102 \\
\hline \multicolumn{3}{|c}{$r_{\max } / r_{\min }$} \\
\hline Frohlich & 4.7 & 3.5 \\
Normal & 4.4 & 2.5 \\
Lognormal & 4.6 & 2.8
\end{tabular}


fits the corresponding range of particle radii within one standard deviation, were determined. Table lists the standard deviation values $\sigma_{\mathrm{n}}, \sigma_{\mathrm{L}}$ (defined in [6]) and the range and ratio of $r_{\max }$ to $r_{\min }$, together with other data obtained for the two samples. It can be seen that in both cases $\sigma_{\mathrm{L}}$ is greater than $\sigma_{\mathrm{n}}$. Furthermore, the Frohlich distribution function gives the largest ratio of $r_{\max } / r_{\min }$, however it must be borne in mind that the Frohlich case represents all the particles in the distribution whilst the corresponding ratios for the other distributions represent only $68 \%$ of the particles.

\section{Conclusion}

From the complex susceptibility measurements both samples were found to have a Debye-type profile, with average particle radii indicative of the presence of aggregation. The aggregates had radii ranging from $36 \mathrm{~nm}$ to $80 \mathrm{~nm}$, far greater than the corresponding mean particle radii of $7 \mathrm{~nm}$ and $6 \mathrm{~nm}$, respectively. Application of Frohlich, normal and lognormal distribution functions has enabled the range of particle aggregates to be determined and compared. In the case of the normal and lognormal distribution functions the range of particle radii were approximately the same, whilst the Frohlich function gave a wider particle range. This difference occurs because of the fact that the Frohlich case represents all the particles in the distribution whilst the corresponding particle range for the other distributions represents the particle sizes within one standard deviation of the distribution. The results obtained illustrate the usefulness of the susceptibility technique for the investigation of aggregates in magnetic fluids.

\section{References}

[1] W.F. Brown, Phys. Rev. 130, 1677 (1963).

[2] W.F. Brown, J. Appl. Phys. 34, 1319 (1963).

[3] P. Debye, Polar Molecules, The Chemical Catalog Company Ltd., New York 1929.

[4] P.C. Fannin, S.W. Charles, J. Magn. Magn. Mater. 72, 95 (1988).

[5] C.J.F. Bottcher, P. Bordewijk, Theory of Electric Polarization, Vol. 2, Elsevier, Amsterdam 1978, p. 86.

[6] K. O'Grady, A. Bradbury, J. Magn. Magn. Mater. 39, 91 (1983).

[7] P.C. Fannin, S.W. Charles, J. Phys. E, Sci. Instrum. 19, 238 (1986). 